\title{
A New Hybrid ARQ-FEC Scheme for Wireless ATM Networks
}

\author{
J. M. C. Brito and I. S. Bonatti
}

\begin{abstract}
Wired ATM (Asynchronous Transfer Mode) networks are designed taking into account an optical link in the physical layer. The main problem to implement a wireless ATM network is the fact that its radio link bit error rate is high and variable, demanding the use of an additional error control mechanism. The usual techniques for error control are ARQ (Automatic Repeat Request) and FEC (Forward Error Correction). ARQ works better than FEC if the channel bit error rate is low, and is worse if it is high, thus indicating that hybrid ARQ-FEC techniques can improve the radio link performance. A new hybrid ARQ-FEC scheme with multiple FEC states is proposed in this paper. The performance of the proposed scheme is compared with non-adaptive ARQ and FEC schemes, which have similar complexities, and with an ideal adaptive FEC scheme. It is shown that the proposed scheme works better than the nonadaptive schemes, and that the hybrid and the ideal adaptive FEC schemes have similar performances. Moreover, the hybrid scheme does not require an estimate of the bit error rate in the channel, leading to a less complex implementation.
\end{abstract}

Index Terms: Wireless ATM Networks, Error Control, Hybrid ARQ-FEC Schemes.

\section{INTRODUCTION}

$\mathrm{B}$ ROADBAND and wireless networks are currently the two major drivers in the telecommunication industry. Asynchronous Transfer Mode (ATM) is the internationally accepted mode for broadband networks and has been used by several telecommunications operators to implement the backbone of their networks. Wireless ATM combines the advantages of the traditional wireless networks with the flexible bandwidth allocation and guaranteed quality of service provided by the wired ATM networks. Moreover, using ATM in the access networks yields the integration of these networks to the backbone of the operators.

Raychaudhuri and Wilson [1] were the first to propose the wireless ATM concept, in 1992. The potential scenarios to use wireless ATM networks are local area networks, residential networks and access networks (with fixed or mobile terminals).

J. M. C. Brito is with National Institute of Telecommunications, P.O. Box 05, S. Rita do Sapucaí, MG, Brazil email: brito@inatel.br

I. S. Bonatti is with School of Electrical \& Computer Engineering, University of Campinas, DT - FEEC - UNICAMP - CP 6101

13081-970, Campinas, SP, Brazil

email: ivanil@dt.fee.unicamp.br
Several research projects have been proposed in the last few years, such as: the WATMnet project, developed by NEC for personal multimedia networks in a micro-cellular environment with fixed and mobile terminals [28]; the Magic Wand (Wireless ATM Network Demonstrator) project, proposed by European Union ACTS (Advanced Communications Technologies and Services), for indoor environment with mobile terminals [2]; the BAHAMA project proposed by Bell Laboratories, for ad hoc local area networks with mobile terminals [3]; the SWAN (Seamless Wireless ATM Networks) developed by Bell Laboratories for indoor networks with picocellular environment and mobile terminals [4]; the ATMmobil project, proposed by a pool of universities and enterprises in Germany, considering four different scenarios: local area networks, mobile broadband cellular networks, residential networks, and fixed access networks [5].

In general, the goal of wireless ATM networks is to provide the same characteristics of the wired ATM networks, in a transparent way, through a wireless link, for fixed or mobile terminals. Thus, wireless ATM networks must integrate several kinds of traffic with guaranteed quality of service. The main problems to make the wireless ATM networks feasible are related to the following research areas [3][6][7]: mobility management, multiple access protocol and error control in the wireless link.

The noisy and time-varying environment of radio links is a problem for wireless ATM networks because wired ATM networks were designed for an optical medium, which has a very low bit error rate. A high bit error rate leads to unacceptable values for the parameters Cell Error Ratio (CER) and Cell Loss Ratio (CLR), requiring additional mechanisms for error control in the radio link. The traditional schemes for error control are Forward Error Correction (FEC) and Automatic Repeat Request (ARQ).

FEC or ARQ in the wireless link decreases the residual bit error rate (i.e., the bit error rate considering the error control mechanism), however the delay in transmitting a cell is increased. The throughput, due to overhead of the error control mechanisms, is also decreased. Therefore, the parameters CTD (Cell Transfer Delay) and CDV (Cell Delay Variation) are affected by the error control mechanism.

There are two different approaches to implement the error control mechanism: a non-adaptive approach, which is simpler and less efficient, and an adaptive approach, which is more complex but can maximize the performance. In non-adaptive schemes, the number of parity bits in the codes used in the FEC or ARQ are calculated for the highest bit error rate in the radio link in order to satisfy the target QoS (Quality of Service) parameters. In adaptive schemes, the number of 
parity bits in the ARQ and FEC codes is function of the bit error rate in the channel, requiring a good estimate of the bit error rate in the wireless link. Moreover, the encoder and decoder must be able to implement codes with different rates.

Several solutions to error control in wireless ATM networks have been proposed in the literature. The techniques used in these solutions include using ARQ limiting the number of retransmissions for delay sensitive applications [2], FEC (with or without concatenated codes) or a hybrid type 1 ARQ-FEC scheme [1][4][5][8]-[12], FEC code to protect the header and another one to protect the payload [13][14], adaptive FEC codes with or without ARQ [13][15]-[18] and FEC code (more powerful than the code specified to the Header Error Control), adaptive or not, to protect the header [19].

The most used ARQ protocols are the Go-Back-N (GBNARQ) [2][8] and the Selective Repeat (SR-ARQ) [1][4][8] (or some variant). The most used FEC codes are the $\mathrm{BCH}$ (Bose, Chaudhuri and Hocquenghem) [1][8][10][13][14][15][19] and the Reed-Solomon [5][8][11]-[13][17]. In the solutions using concatenated codes, the most common combination is a ReedSolomon or BCH code with a convolutional code [10]-[12].

A new hybrid ARQ-FEC scheme that has multiple FEC states is proposed in this paper. The performance of the proposed scheme is compared with non-adaptive FEC and ARQ schemes (all having similar complexity). It is also compared with an ideal adaptive scheme, used as reference. The proposed scheme is proved to work better than the nonadaptive schemes and it has similar performance when compared with the ideal adaptive FEC scheme.

The reminder of this paper is organized as follow: the reference scenario used in the analysis is presented in Section 2; in Section 3, the criterion to define the codes is presented; in Section 4, the non-adaptive ARQ and FEC schemes and an ideal FEC scheme are analyzed; in Section 5, the scheme proposed is presented and its performance is computed; conclusions are presented in Section 6; a summary of the notations used in the text is presented in the Appendix.

\section{REFERENCE SCENARIO}

The reference scenario is composed of one wireless and $L$ optical links. In the wireless link, several terminals share a radio-base station using a multiple access protocol. The error control mechanism and the multiple access protocol are implemented in a Data Link Layer (usually comprising a Data Link Control Layer and Multiple Access Control Sub-Layers) located between the ATM and Physical Layers.

The main multiple access technique used in the wireless ATM networks reported in the literature is a TDMA/TDD (Time Division Multiple Access / Time Division Duplex) technique [1][2][8][15]. A DAMA-TDMA (Demand Allocation Multiple Access - Time Division Multiple Access) multiple access is considered in this paper. In this scheme, the time is divided into frames, each frame being divided in downlink and uplink periods. In the downlink period, the basestation transmits to terminals using Time Division Multiplexing (TDM). The uplink period is divided in a contention period and a transmission period. The terminals send requests in the contention period using a random multiple access (like Slotted-Aloha) and, when allowed, transmit data in the transmission period using TDMA. The transmission period is divided into slots, each transmitting one ATM cell.

An ATM cell consists of a 40-bit header and a 384-bit information field. The header includes an 8-bit header error control (HEC) field. The HEC has two operation modes: correction mode and detection mode. In the correction mode the HEC can correct 1-bit errors. The ATM adaptation layer (AAL) considered for data transmission is the AAL5 [27]. Interleaving is used to randomize channel burst errors in such way that a memoryless channel model can be considered. The frames and their periods in the wireless link are illustrated in Fig. 1 .

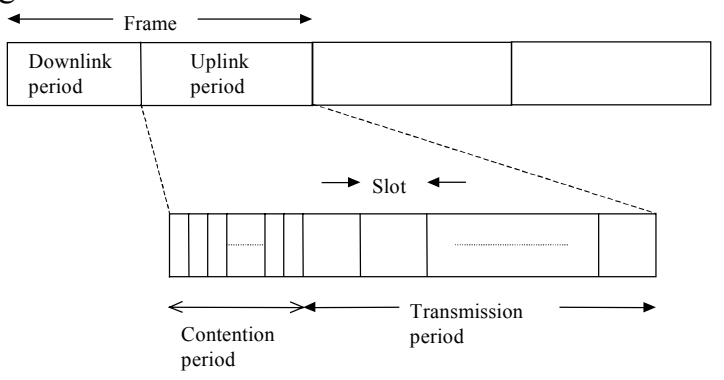

Fig. 1. Frame structure in the wireless link.

Downlink and uplink periods are considered to have the same fixed size slots. The size of the slots in the reservation period can be represented as a fraction of the size of the slots in the transmission period. Thus, the frame time can be computed by

$$
T_{q}=\frac{V_{u} \cdot \tau+V_{d} \cdot \tau+\alpha \cdot V_{u} \cdot \tau}{R_{s}}=\frac{V \cdot \tau}{R_{s}}
$$

where $V_{u}$ and $V_{d}$ represent the number of slots in the uplink and downlink periods, respectively; $\alpha$ represents the size of the slots in the reservation period as a fraction of the slots in the transmission period; $R_{s}$ is the bit rate in the wireless link; $\tau$ is the total number of bits in each transmission slot in the uplink period, and $V=V_{u}+V_{d}+\alpha V_{u}$. The parameter $\tau$ depends on the error control technique used in the wireless link.

The time delays that affect all the systems in the same way have not been considered in the analysis. Therefore, the time to make a reservation, the overheads of the MAC (Medium Access Control) protocol, the wired network delays (like queuing and switching time delays), and the time spent to transmit an acknowledgement from the receiver to the transmitter are not taken into account.

\section{DEFINING THE ARQ AND FEC CODES}

The criterion used to define the ARQ and FEC codes is to make the wireless link work like an optical link, concerning the bit error characteristic. This is equivalent to say that the probability of the wireless link to deliver a correct cell to the optical link is equal or greater than the probability of one optical link to deliver a correct cell to the next optical link. This criterion is in agreement with the analysis presented by Cain and McGregor in [11]. 
Considering that the HEC is in the correction mode, the probability of an optical link to hand a correct cell to the next optical link is the probability of the cell to contain no errors in the payload field and to contain at most 1-bit errors in the header. This probability is given by

$$
P_{c c o}=(1-p)^{384} \cdot\left[(1-p)^{40}+40(1-p)^{39} \cdot p\right]
$$

where $p$ is the bit error rate. The value of the parameter $p$ was considered as $10^{-8}$ for optical links.

\section{A. CHOICE OF ARQ CODES}

In ARQ techniques, the cells are individually protected by an $(n, k)$ block code, being $k$ the number of information bits, $n$ the total number of bits and $(n-k)$ the number of parity bits. One cell arriving in the base station can contain no errors with probability $P_{c}$, it can contain a detectable error with probability $P_{e}$, or it can contain an undetectable error with probability $P_{u e}$. The probability of a cell to leave the radio link without error is given by [25]

$$
P_{c c a}=P_{c}+P_{c} \cdot \sum_{k=1}^{\infty} P_{e}^{k}=\frac{P_{c}}{1-P_{e}}=\frac{P_{c}}{P_{c}+P_{u e}}
$$

Considering that the HEC of the ATM header is not used in the error control mechanism in the wireless link, the probability $P_{c}$ is computed by

$$
P_{c}=(1-p)^{n}
$$

To satisfy the criterion, the maximum permitted value for $P_{u e}$ is given by

$$
P_{u e} \leq \frac{P_{c} \cdot\left(1-P_{c c o}\right)}{P_{c c o}}
$$

The minimum number of parity bits in the ARQ codes can be computed using an iterative algorithm and the Korzhik's bound, given by the expression [20]

$$
P_{u e} \leq 2^{-(n-k)}\left[1-(1-p)^{n}\right]
$$

\section{B. CHOICE OF THE FEC CODES}

In the FEC technique, an $(n, k)$ block code is used to correct errors in each cell. Not considering the HEC influence, the probability of the decoder to deliver a correct cell to the optical link is given by

$$
P_{c c f}=\sum_{i=0}^{t}\left(\begin{array}{l}
n \\
i
\end{array}\right) \cdot p^{i} \cdot(1-p)^{n-i}
$$

The parameter $t$, which is the error correction capacity of the FEC code, is a function of the $n-k$ value and of the code used. To compute the minimum number of parity bits in FEC codes, we have used an iterative algorithm and the Plotkin's bound, given by [21]

$$
n-k \geq 2\left(d_{\min }-1\right)-\log _{2} d_{\min }
$$

where $d_{\min }$ is the minimum distance of the code.

\section{COMPARING ARQ AND FEC PERFORMANCES}

The time delay to transmit a PDU (Protocol Data Unit), composed of $Z$ ATM cells, was adopted as the parameter to compare the performance of ARQ and FEC schemes.

The mean time to transmit a correct PDU in a system using Stop-and-Wait ARQ (SW-ARQ) protocol in the wireless link, not considering the time to transmit the acknowledgement from destination to the source terminal, can be calculated by [22]

$$
T_{s w a}=\left[T_{q} \cdot\left(\frac{Z}{P_{c}}-1\right)+\frac{n}{R_{s}}+\xi_{a}+\frac{424 L}{R_{o}}\right] \cdot \gamma
$$

where $T_{q}$ is the frame time, computed by (1), $\xi_{a}$ is the decoding delay of the ARQ code, $R_{s}$ and $R_{o}$ are the bit rate in the wireless and optical links, respectively, and $\gamma$ is the mean number of transmissions needed to receive a correct PDU, considering retransmission in the AAL layer.

The parameter $\gamma$ can be calculated by

$$
\gamma=\sum_{i=1}^{\infty} i \cdot P_{p c} \cdot\left(1-P_{p c}\right)^{i-1}=\frac{1}{P_{p c}}
$$

where $P_{p c}$, the probability to receive a correct PDU, is given by

$$
P_{p c}=P_{c c o}{ }^{(L+1) Z}
$$

For a FEC system, in which one cell is transmitted on each slot, the mean time to transmit a correct PDU is given by [22]

$$
T_{f e c}=\left[(Z-1) \cdot T_{q}+\frac{n}{R_{s}}+\frac{424 \cdot L}{R_{o}}+\xi_{f}\right] \cdot \gamma
$$

where $\xi_{f}$ is the decoding delay of the FEC code.

As several parameters affect both techniques in the same way, one can simplify the computation defining a performance factor, $\delta$, as the ratio between the time delay to transmit a PDU in the FEC scheme and the same delay in the ARQ scheme.

Considering that the bit rate in the wireless link and optical links are the same and that the codes are implemented in hardware (the decoding delays are negligible), the performance factor comparing Stop-and-Wait ARQ and FEC is given by

$$
\delta_{s w}=\frac{V \cdot(Z-1) \cdot n_{f}+n_{f}+424 L}{V \cdot n_{s w} \cdot\left(\frac{Z}{P_{c}}-1\right)+n_{s w}+424 L}
$$

where the sub-index $f$ and $s w$ are included in some parameters to determine if the parameter is related to the FEC or SWARQ technique, respectively.

The parameters $L, V$ and $Z$ have negligible influence in the performance factor value. The total number of bits in the FEC and ARQ codes, parameters $n_{f}$ and $n_{s w}$, respectively, are computed to achieve the criterion presented in Section 3. For non-adaptive implementation, $n_{f}$ and $n_{s w}$ are computed considering the highest error rate in the channel, resulting in $n_{f}$ $=487$ bits and $n_{s w}=449$ bits.

Fig. 2 shows the performance factor $\delta$ as a function of the bit error rate in the wireless channel for non-adaptive system 
considering $L=3, V=40$, and $Z=5$ or $Z=1000$. The ARQ technique has better performance than the FEC technique if the bit error rate is low and worse if the bit error rate is high. Both techniques have the same performance for $p=1.8 \times 10^{-4}$.

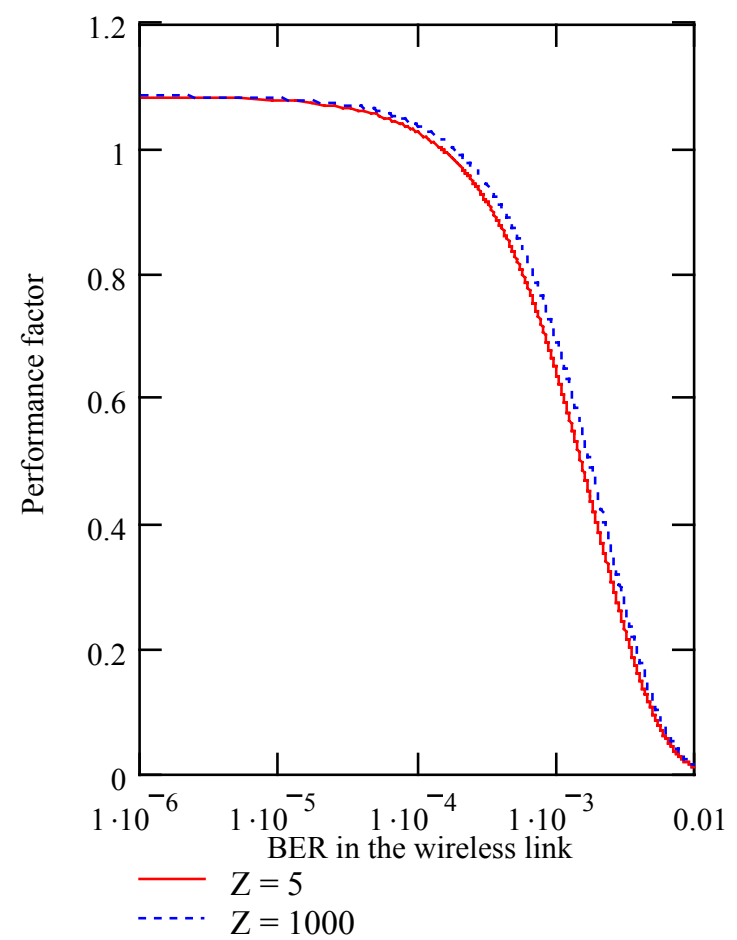

Fig. 2. Performance factor comparing SW-ARQ and FEC schemes.

When comparing the Go-Back-N and Selective-Repeat ARQ protocols with the FEC scheme, the conclusion is similar to the conclusion obtained for the Stop-and-Wait protocol. The ARQ scheme performs better than the FEC scheme if the bit error rate is low and worse if the bit error rate is high. A detailed comparison among these techniques can be found in [22][23][24].

For an adaptive implementation, the parameters $n_{f}$ and $n_{s w}$ are calculated as a function of the bit error rate in the channel. Fig. 3 shows the performance factor comparing ARQ techniques and the FEC technique considering an ideal adaptive implementation, for which the estimate of the bit error rate in the channel can be obtained without additional overhead. The parameters $L, V$ and $Z$ were fixed to the following values: $L=3, V=40$ and $Z=100$. For the GoBack-N and Selective-Repeat protocols, $N$ was set as $N=5$ [22].

With ideal adaptive implementation, the FEC technique works better than the ARQ techniques in the whole range of bit error rate considered.

Based on the results presented in this section, it can be concluded that the FEC adaptive scheme maximizes the performance of the network. However, an adaptive implementation increases the complexity and, in real world, it can require additional overhead, decreasing the efficiency of the system. If, as an attempt to reduce the overhead, the estimate is made only at the start of the connection, changes in the behaviour of the wireless link can result in a significant degradation of the performance.

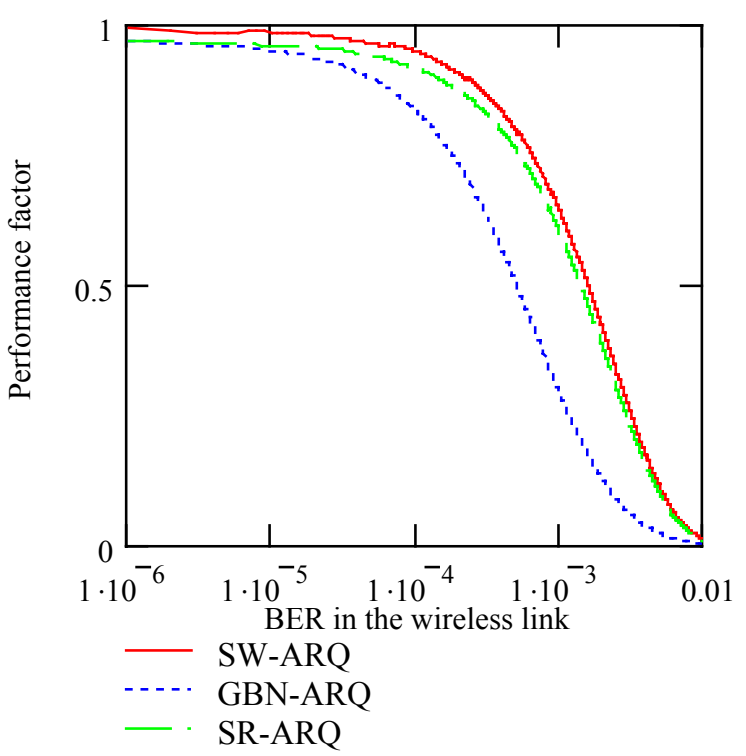

Fig. 3. Performance factor comparing ARQ and FEC schemes, considering adaptive codes.

If a non-adaptive scheme is considered, the results in Fig. 2 show that a hybrid ARQ-FEC technique can improve the performance of the network.

In the following sections, the ideal adaptive FEC scheme is used as a reference to analyze the performance of the proposed techniques.

\section{HYBRID ARQ-FEC SCHEME}

A hybrid adaptive ARQ-FEC technique, which does not require an estimate of the bit error in the channel, is presented in this section. In this technique there are two codes: one for correction (FEC code) and one for detection (ARQ code). The total number of bits (424 bits of the ATM cell plus the parity bits) of the FEC code is defined as $n_{f}$ and the total number of bits of the ARQ code is defined as $n_{a}$. Since there is not an estimate of the bit error rate in the channel, the number of parity bits of the FEC and ARQ codes must be chosen considering the worst bit error rate, $p=10^{-2}$.

In this scheme, the system switches to the FEC mode when a cell containing error is received (and the retransmission is requested), and switches to the ARQ mode when a correct cell is received, as shown in Fig. 4 [25].

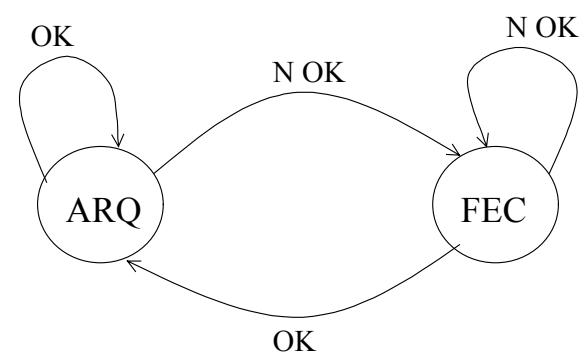

Fig. 4. Transition diagram.

The probability of the wireless link to deliver a correct cell to the optical link is given by [25] 


$$
P_{c c h}=\left(P_{c}+P_{e} \cdot P_{c c f}\right) \cdot P_{a r q}+P_{c c f} \cdot\left(1-P_{a r q}\right)
$$

where $P_{c}$ is the probability of a cell to contain no errors in the ARQ state, $P_{e}$ is the probability of a cell to contain a detectable error in the ARQ state, $P_{e}=1-P_{c}-P_{u e}, P_{c c f}$ is the probability of a cell to contain a correctable error in the FEC state, given by (7), and $P_{a r q}$ is the steady state probability of the ARQ state, given by [25]

$$
P_{\text {arq }}=\frac{P_{c f}}{P_{c f}+P_{e}}
$$

where $P_{c f}$ is the probability of a cell to contain no errors in the FEC state. The probabilities $P_{c}$ and $P_{c f}$ are calculated by (4).

The minimum number of parity bits for each code can be obtained as follows. First set $p$ as the highest bit error rate in the channel (for example, $p=10^{-2}$ ). Then, setting $n_{a}$ big enough to make $P_{u e}$ negligible, $P_{a r q}$ tends to zero e $P_{c c h}$ tends to $P_{c c f}$ and the minimum number of parity bits of the FEC code can be computed using the approach presented in Section 3. As shown in Fig. 5, the FEC code requires at least $t=17$ and it is not possible to decrease this bound even by increasing indefinitely the number of parity bits in the ARQ code. Also, one can verify that $n_{a} \geq 438$ to attain $P_{c c h} \geq P_{c c o}$. Thus, for $p=$ $10^{-2}$, the minimum number of parity bits of the ARQ and FEC codes are $14\left(n_{a}=438\right)$ and $63\left(n_{f}=487\right)$, respectively.

When the bit error rate decreases, the probability of the system being in the FEC state also decreases, and the influence of the ARQ code in $P_{c c h}$ value increases. In a first approach, the minimum number of parity bits of the ARQ code can be obtained setting the minimum value for $n_{f}$ and computing the value of $n_{a}$ for each value of $p$, in such way that $P_{c c h} \geq P_{c c o}$. As shown in Fig. 6, the ARQ code requires 16 parity bits $\left(n_{a}=440\right.$ bits) to achieve $P_{c c h} \geq P_{c c o}$ in the whole bit error rate range considered.

The probability $P_{c c h}$ depends on the parameters $n_{f}$ and $n_{a}$. However, it is not possible to reduce the value of $n_{a}$ by increasing the value of $n_{f}$, as shown in Fig. 7. Therefore, the final solutions for the codes are $n_{a}=440$ bits and $n_{f}=487$ bits.

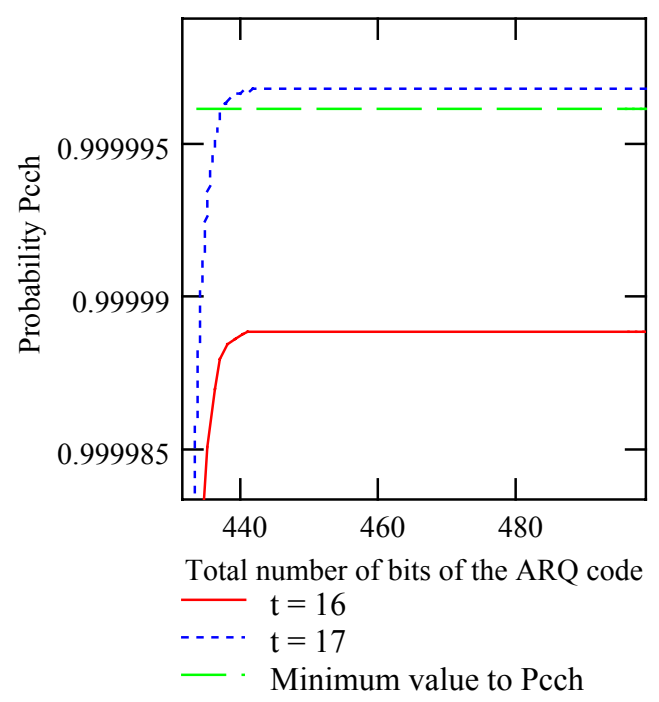

Fig. 5. Minimum capacity of the FEC code, for $p=10^{-2}$.

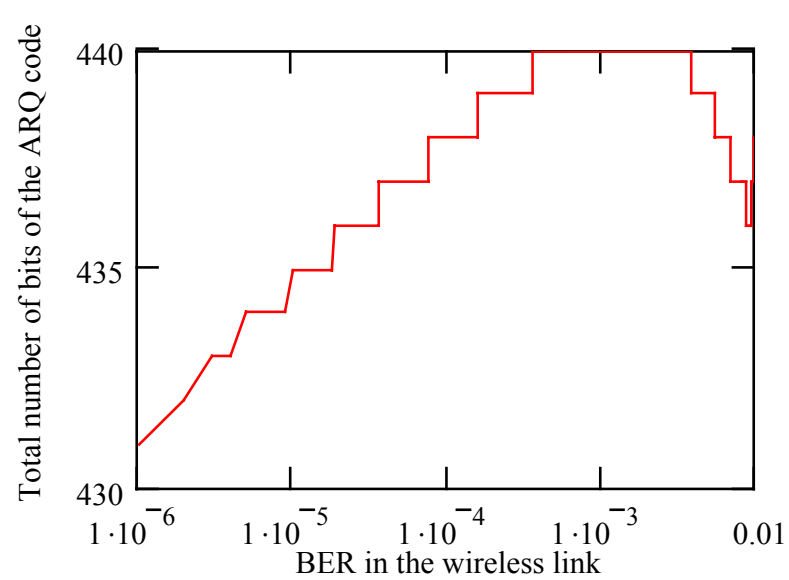

Fig. 6. Total number of bits of the ARQ code, as a function of $p$, for $n_{f}=487$.

The probability $P_{c c h}$ depends on the parameters $n_{f}$ and $n_{a}$. However, it is not possible to reduce the value of $n_{a}$ by increasing the value of $n_{f}$, as shown in Fig. 7. Therefore, the final solutions for the codes are $n_{a}=440$ bits and $n_{f}=487$ bits.

The mean time to transmit the first $(Z-1)$ cells of the PDU, considering that all slots in the frame are in the same state, can be computed by [25]

$$
T_{z-1}=\left[T_{q a}\left(1-P_{e}\right)+\left(T_{q a}+T_{q f}\right) P_{e}\right] P_{a r q}+T_{q f}\left(1-P_{a r q}\right)
$$

where $T_{q a}$ and $T_{q f}$ are the frame time of the ARQ and FEC modes, respectively, and can be computed using (1), setting the $n$ value properly.

The mean time to transmit the last cell of the PDU is given by [25]

$$
T_{z}=\left[T_{x a}\left(1-P_{e}\right)+\left(T_{q a}+T_{x f}\right) \cdot P_{e}\right] P_{a r q}+T_{x f}\left(1-P_{a r q}\right)
$$

where $T_{x a}$ and $T_{x f}$ are the time to transmit one cell in the ARQ state and FEC state, respectively, and can be computed dividing the number of bits in the transmission slot, in the proper state, by the bit rate in the wireless link.

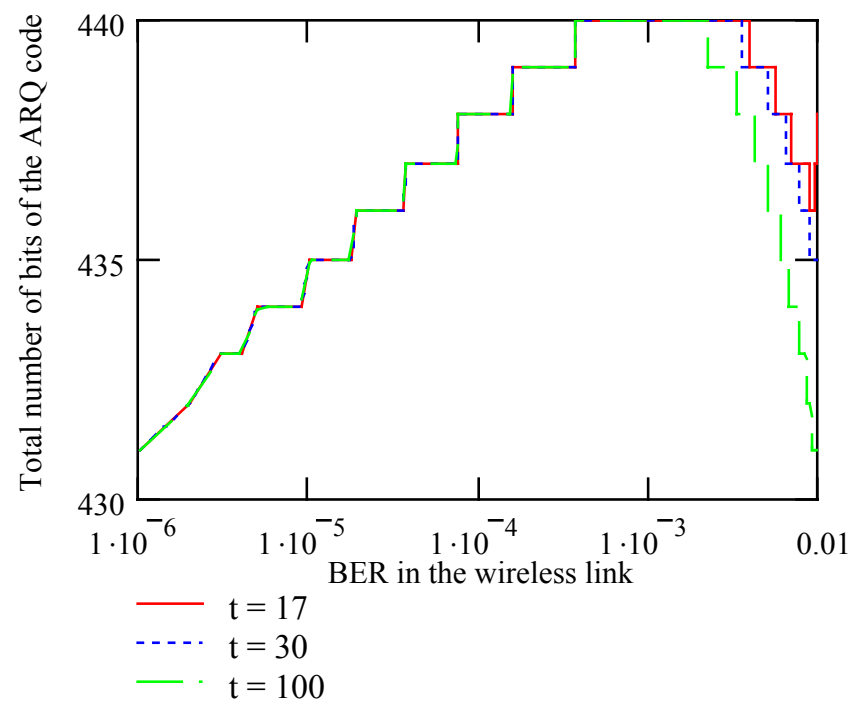

Fig. 7. Influence of the value of $t$ in the total number of bits of the ARQ code, as a function of $p$. 
The mean time to transmit a correct PDU, not considering the time to transmit the acknowledgement from the destination to the source terminal, is given by [25]

$$
T_{h}=\left[(Z-1) \cdot T_{z-1}+T_{z}+\frac{424 L}{R_{o}}\right] \cdot \gamma
$$

where $\gamma$, the mean number of transmissions needed to receive a correct PDU, is computed by (9) and (10).

The throughput for the hybrid ARQ-FEC technique is computed by

$$
\eta_{h}=\frac{k}{\left[n_{a} \cdot\left(1-P_{e}\right)+\left(n_{a}+n_{f}\right) \cdot P_{e}\right] \cdot P_{a r q}+n_{f} \cdot\left(1-P_{a r q}\right)}
$$

To assess the performance of the hybrid ARQ-FEC technique, its performance has been compared to that of the non-adaptive SW-ARQ and FEC techniques, which have similar complexities. Two performance factors have been defined. The first one is defined as the ratio between the mean time to transmit a correct PDU in the SW-ARQ technique (given by (9)) and the corresponding time in the hybrid technique (given by (18)). The second one is defined as the ratio between the mean time in the FEC technique (given by (12)) and the corresponding time in the hybrid scheme. Figures 8 and 9 show the results, as a function of $p$, considering $V=40, L=3$ and $Z=100$.

The hybrid scheme performs better than the non-adaptive SW-ARQ technique in the whole range of the bit error rate considered and the difference between the two techniques is more significant for high bit error rates. The hybrid scheme has better performance than the non-adaptive FEC technique only for $p \leq 2.310^{-4}$, but the decline in performance for higher bit error rate is not so significant.

A performance comparison between the hybrid scheme and the ideal adaptive FEC scheme is shown in Fig. 10, based on the mean time to transmit a correct PDU, and in the Fig. 11, based on the throughput (the throughput is $k / n$ in the FEC scheme). The performance factor, presented in Fig. 10, is defined as the ratio between the time to transmit a correct PDU in the FEC scheme and the corresponding time in the hybrid scheme. The hybrid and the ideal adaptive FEC schemes have similar performance, except for $p$ around $10^{-3}$.

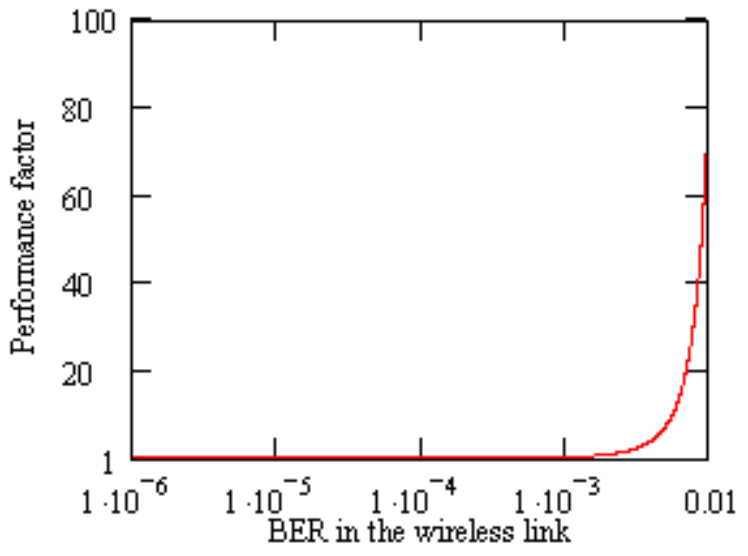

Fig. 8. Performance factor comparing the non-adaptive SW-ARQ and the hybrid schemes.

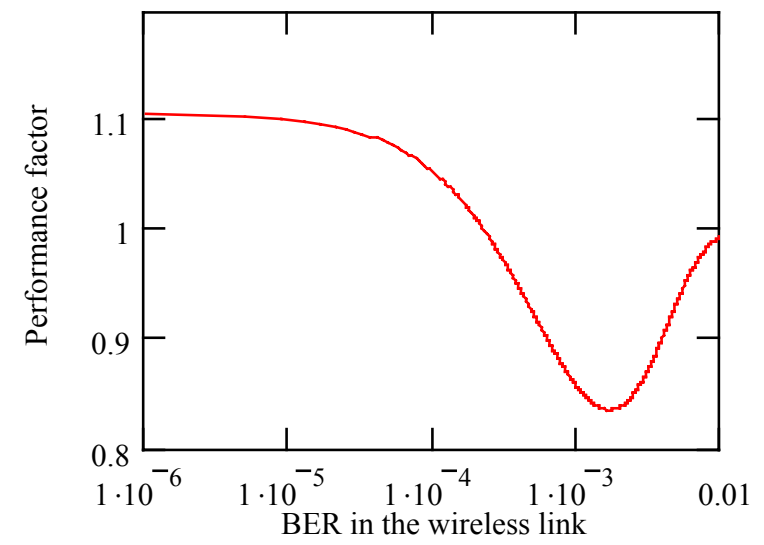

Fig 9. Performance factor comparing the non-adaptive FEC and the hybrid schemes.

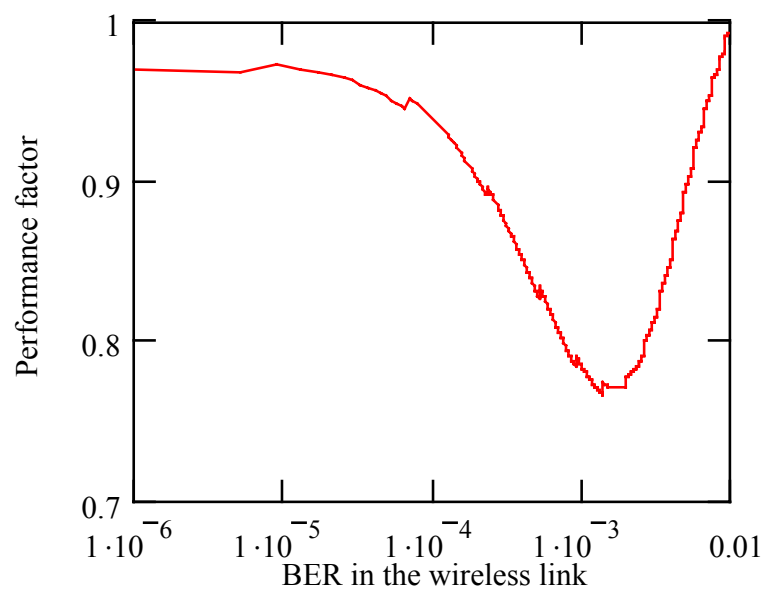

Fig. 10. Performance factor comparing the hybrid and the ideal adaptive FEC schemes.

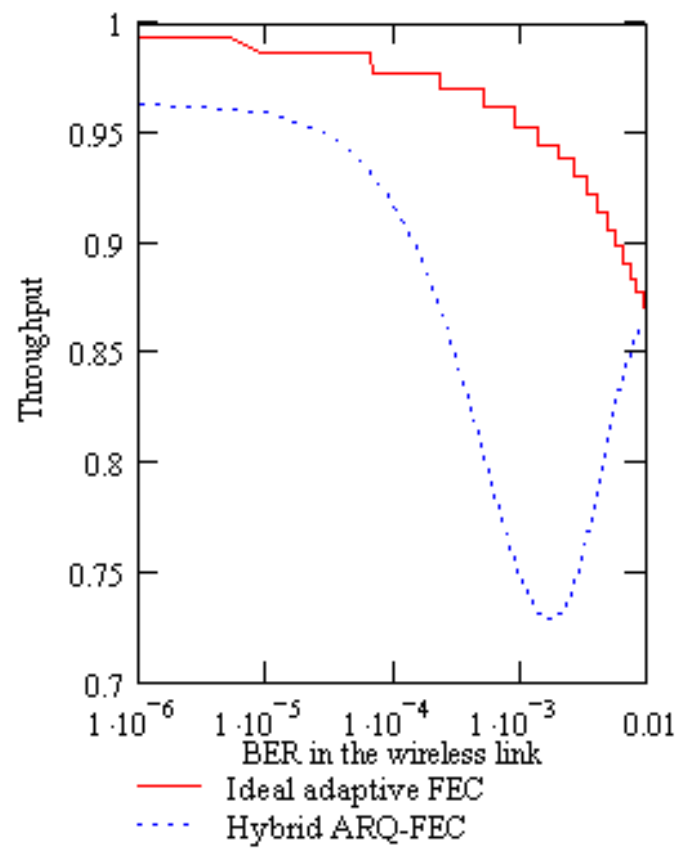

Fig. 11. Comparing the throughput in the hybrid scheme and in the ideal adaptive FEC scheme. 
The "valley" in the hybrid scheme's performance, around $p=10^{-3}$, occurs due to the large probability of switching between ARQ and FEC states for those values of $p$. Fig. 12 shows the probability of the ARQ and FEC states as a function of $p$.

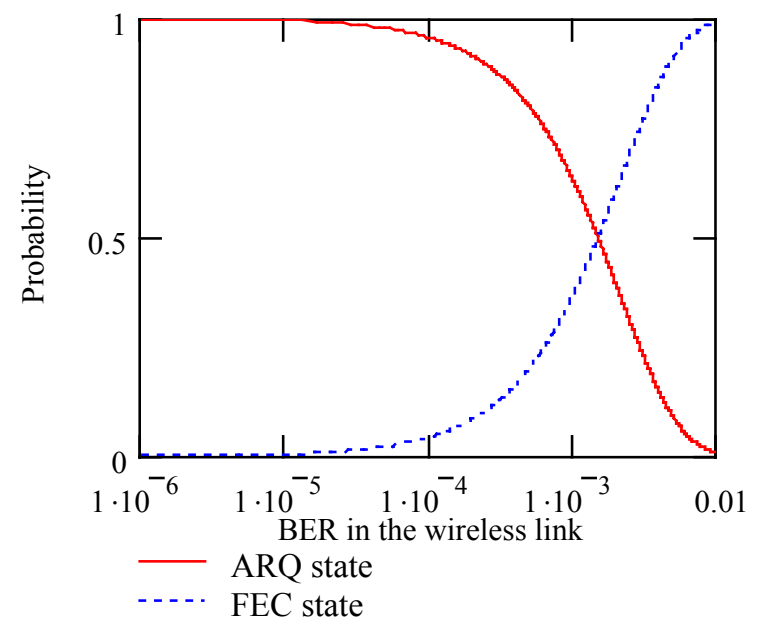

Fig. 12. Probability of the ARQ and FEC states.

\section{A. HYBRID ARQ-FEC SCHEME WITH MULTIPLE FEC STATES}

Since the FEC technique has better performance than the ARQ technique when the bit error rate in the channel is high, the performance of the hybrid ARQ-FEC scheme can be improved by increasing the probability of the system to be in the FEC state for high bit error rates. To achieve this behaviour, an approach with multiple FEC states, in which the switching from the FEC to the ARQ state occurs only after receiving $y$ consecutive correct cells is proposed. The state diagram of this new system is shown in Fig. 13 [26].

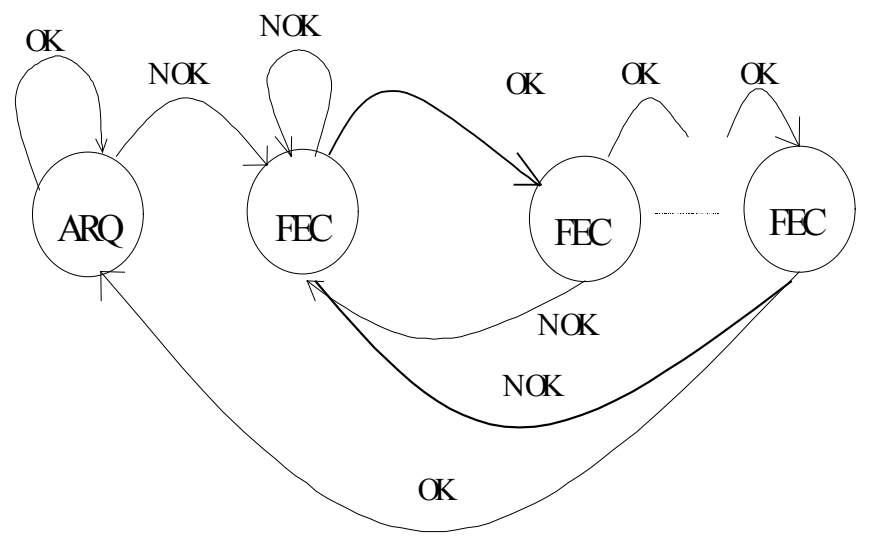

Fig. 13. State diagram for the hybrid approach with multiple FEC states.

The probability of the ARQ state can be written as [26]

$$
P_{\text {arq }}=\frac{P_{c f}{ }^{y}}{P_{c f}{ }^{y}+P_{e} \cdot \sum_{j=0}^{y-1} P_{c f}{ }^{j}}
$$

Fig. 14 shows the influence of the $y$ value in the probabilities of the ARQ and FEC states, considering $n_{a}=440$ and $n_{f}=487$ bits. It can be seen that the value of $p$ in the crosspoint decreases when $y$ increases.

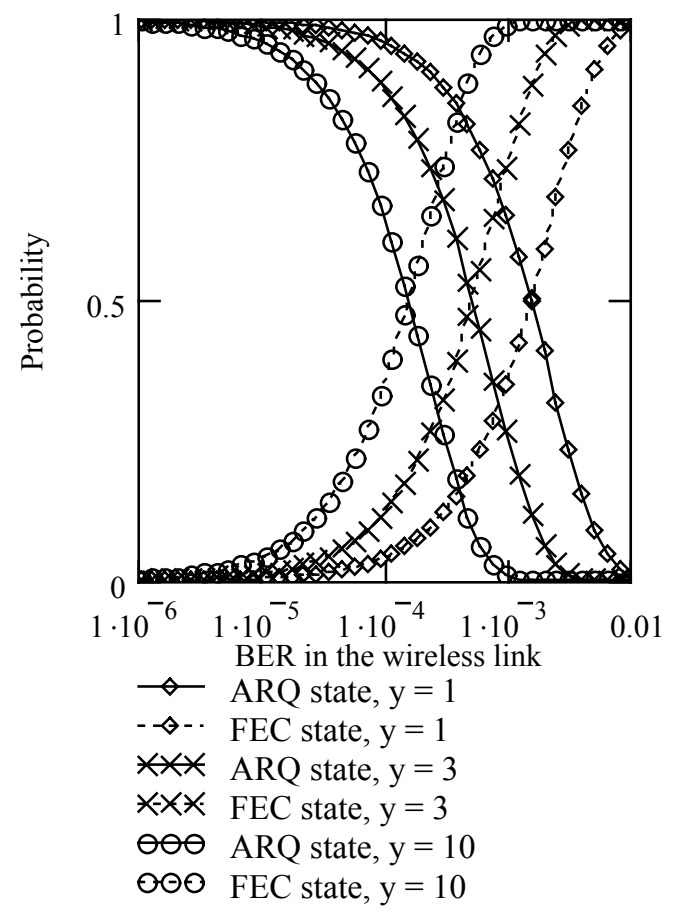

Fig. 14. Probability of the ARQ and FEC states, as a function of $y$.

The ARQ and FEC codes must be defined such that $P_{c c h} \geq$ $P_{c c o}$, with the $P_{c c h}$ value computed by expression (14), using $P_{a r q}$ given by expression (20).

Setting $p=10^{-2}$, the largest bit error rate in the channel, and choosing $n_{a}$ big enough to make $P_{u e}$ insignificant, $P_{c c h}$ tends to $P_{c c f}$, independently of the $y$ value, and the minimum capacity of the FEC code can be found. The minimum capacity is $t=$ 17, resulting in $n_{f}=487$ bits, as shown in Fig. 5 .

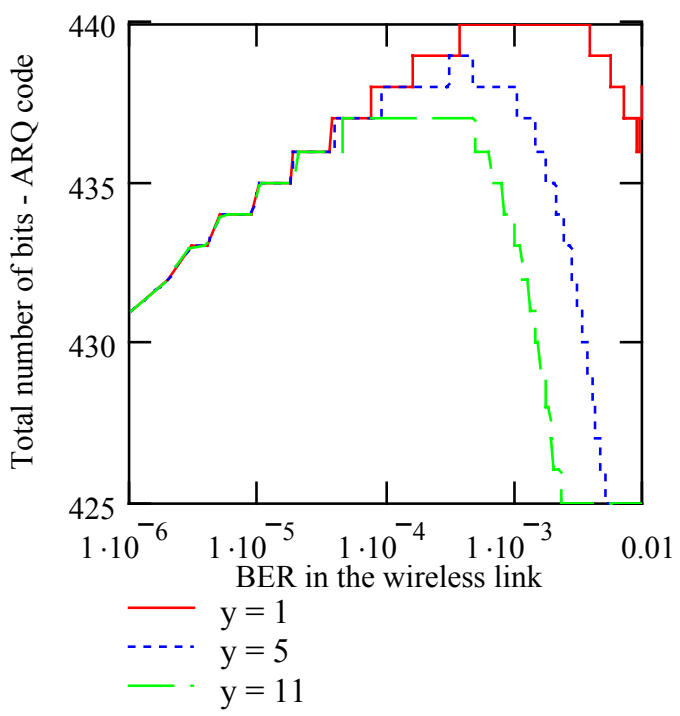

Fig. 15. Minimum total number of bits of the ARQ code. 
If $n_{f}$ is equal to its minimum value, the number of parity bits of the ARQ code that produce $P_{c c h} \geq P_{c c o}$ can be computed. Fig. 15 shows the results for $y=1\left(n_{a}=440\right), y=5$ $\left(n_{a}=439\right)$ e $y=11\left(n_{a}=437\right)$ [26]. Since $P_{c c h}$ depends on $n_{f}$ and $n_{a}$, it is necessary to investigate if it is possible to reduce the number of parity bits in the ARQ code by increasing the capacity of the FEC code beyond its minimum value. The capacity of the FEC code has been varied, starting with $t=17$ and, for each value of $t$, the minimum value of $n_{a}$ has been computed for each value of $p$ such that $P_{c c h} \geq P_{c c o}$. Fig. 16 shows the results for $t=17, t=30$ and $t=100$, considering $y=$ 5. Although a big value for $t$ results in a small $n_{a}$ for some values of $p$, it is still necessary to have $n_{a} \geq 439$, for $y=5$, to achieve $P_{c c h} \geq P_{c c o}$ for any values of $p$. The conclusions are the same for other values of $y$ [26].

The mean time to transmit a correct PDU is calculated by expression (18), with $P_{\text {arq }}$ being given by expression (20). Fig. 17 compares the performance between the hybrid scheme and a non-adaptive FEC scheme, assuming $R_{s}=R_{o}, L=3, V=40$ and $Z=100$.

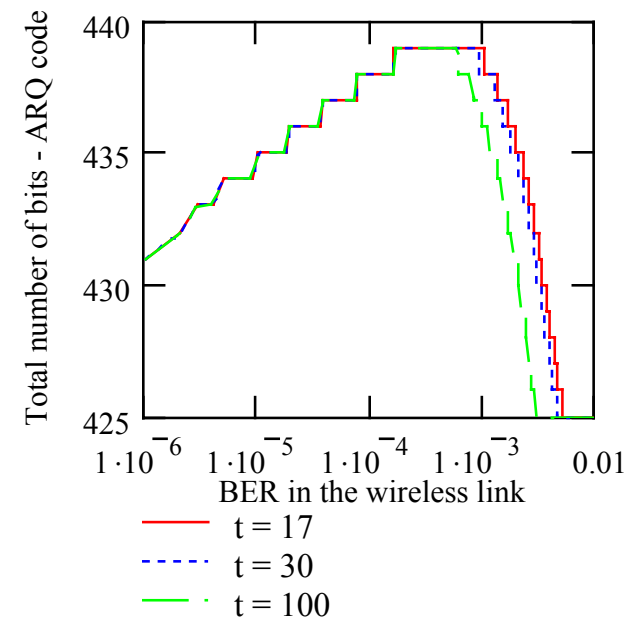

Fig. 16. Minimum total number of bits of the ARQ code, as a function of $t$.

The "valley" in the performance factor tends to disappear when $y$ increases. For $y=11$, the "valley" practically disappear, without significant loss of performance for low bit error rates. Thus, by properly choosing $y$, the new hybrid ARQ-FEC scheme performs better than the older one $(y=1)$ in the whole range of $p$.

The new hybrid scheme has similar performance to the ideal adaptive FEC scheme as shown in Fig. 18. The hybrid scheme does not require an estimate of the bit error rate in the channel, resulting in a non-complex implementation.

The throughput in the hybrid scheme is given by expression (19), with $P_{a r q}$ being computed by expression (20). The throughput of the hybrid scheme can be maximized by properly choosing $y$, as shown in the Fig. 19. Again, for $y=$ 11 , the "valley" practically disappear, without significant loss of performance for low bit error rates. Moreover, the throughputs in the hybrid and in the ideal adaptive FEC schemes are similar.

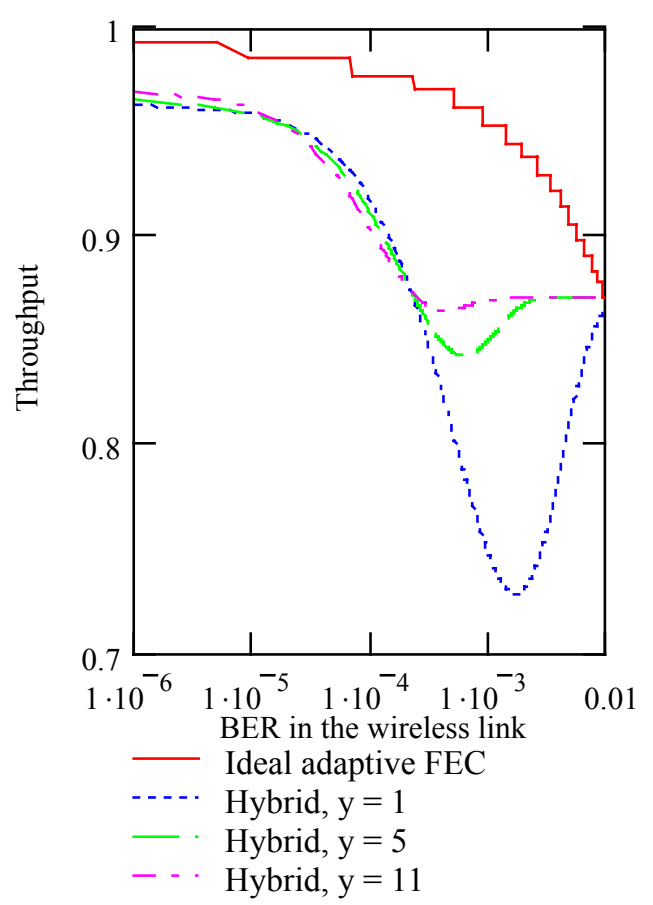

Fig. 17. Performance factor comparing the hybrid and the non-adaptive FEC schemes.

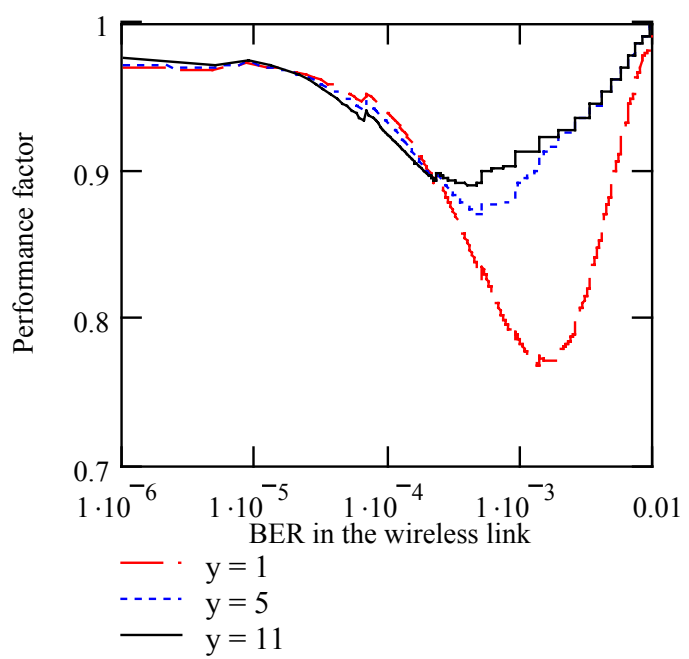

Fig. 18. Performance factor comparing the ideal adaptive FEC and the new hybrid ARQ-FEC schemes.

\section{CONCLUSIONS}

A new hybrid ARQ-FEC scheme with multiple FEC states has been proposed in this paper. To compare the performance of the error control schemes, the mean time to transmit a correct PDU (composed by $Z$ cells) and the throughput in the network were analytically computed. 


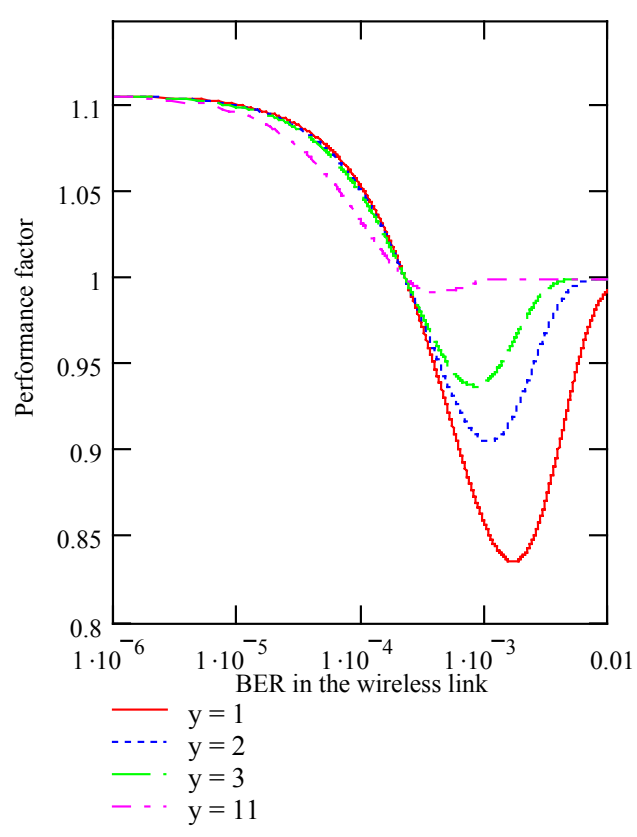

Fig. 19. Comparing the throughput in the hybrid ARQ-FEC scheme and in the ideal adaptive FEC scheme.

The proposed scheme and two non-adaptive ARQ and FEC schemes (all of them having similar complexities) have been compared with an ideal adaptive FEC scheme (considered as a reference). It has been shown that the proposed scheme performs equal or better than the non-adaptive ARQ and FEC schemes provided that the number of FEC states is properly chosen. Moreover, although the ideal adaptive FEC and the hybrid schemes have similar performances, the latter does not need an estimate of the channel bit error rate, thus having low implementation complexity.

\section{APPENDIX:}

\section{SUMMARY OF THE MAIN PARAMETERS}

\begin{tabular}{|l|l|}
\hline$L$ & number of optical links in the network \\
\hline$V$ & total number of slots in a frame \\
\hline$R_{s}$ & bit rate in the wireless link \\
\hline$T_{q}$ & frame time \\
\hline$P_{c c o}$ & $\begin{array}{l}\text { probability of an optical link to hand a correct cell } \\
\text { to the next optical link }\end{array}$ \\
\hline$p$ & bit error rate \\
\hline$k$ & number of information bits in a block code \\
\hline$n-k$ & number of parity bits in a block code \\
\hline$n$ & total number of bits in a block code \\
\hline$P_{c}$ & probability of a cell to contain no errors \\
\hline$P_{e}$ & probability of a cell to contain a detectable error \\
\hline$P_{u e}$ & probability of a cell to contain an undetectable error \\
\hline$P_{c c f}$ & $\begin{array}{l}\text { probability of the FEC decoder to deliver a correct } \\
\text { cell to the optical link }\end{array}$ \\
\hline$t$ & error correction capacity of the FEC code \\
\hline$Z$ & number of ATM cells in a PDU \\
\hline$R_{o}$ & bit rate in the optical links \\
\hline$\gamma$ & mean number of transmissions needed to receive a \\
\hline
\end{tabular}

\begin{tabular}{|l|l|}
\hline & correct PDU \\
\hline$\delta$ & $\begin{array}{l}\text { performance factor - the ratio between the time } \\
\text { delay to transmit a PDU in a FEC scheme and the } \\
\text { same delay in an ARQ scheme }\end{array}$ \\
\hline $\mathrm{n}_{\mathrm{f}}$ & total number of bits in the FEC code \\
\hline $\mathrm{n}_{\mathrm{a}}$ & $\begin{array}{l}\text { total number of bits of the ARQ code in the hybrid } \\
\text { scheme }\end{array}$ \\
\hline $\mathrm{P}_{\mathrm{cch}}$ & $\begin{array}{l}\text { probability of the wireless link to deliver a correct } \\
\text { cell to the optical link in the hybrid scheme }\end{array}$ \\
\hline $\mathrm{P}_{\text {arq }}$ & $\begin{array}{l}\text { steady state probability of the ARQ state in the } \\
\text { hybrid scheme }\end{array}$ \\
\hline $\mathrm{P}_{\mathrm{cf}}$ & $\begin{array}{l}\text { probability of a cell to contain no errors in the FEC } \\
\text { state in the hybrid scheme }\end{array}$ \\
\hline $\mathrm{y}$ & $\begin{array}{l}\text { number of consecutive correct cells producing a } \\
\text { transition from FEC state to ARQ state in the } \\
\text { hybrid scheme }\end{array}$ \\
\hline
\end{tabular}

\section{ACKNOWLEDGMENT}

The authors wish to thank the financial support of the Conselho Nacional de Desenvolvimento Científico e Tecnológico - CNPq, Brazil.

\section{REFERENCES}

[1] D. Raychaudhuri and N. Wilson, Multimedia Personal Communication Networks: System Design Issues, Proc. $3^{\text {rd }}$ Workshop Third Generation Wireless Inform. Networks, Rutgers University, New Brunswick, April 1992, pp. 259-288.

[2] J. Mikkonen, J. Aldis, G. Awater, A. Lunn and D. Hutchison, The Magic WAND - Functional Overview, IEEE Journal on Selected Areas in Communications, Vol. 16, No 6, pp. 953-972, August, 1998.

[3] E. Ayanoglu, K. Y. Eng and M. J. Karol, Wireless ATM: Limits, Challenges, and Proposals, IEEE Personal Communications, pp. 18-33, August, 1996.

[4] P. Agrawal et alii, SWAN: A Mobile Multimedia Wireless Network, IEEE Personal Communications, pp. 18-33, April, 1996.

[5] R. Keller et alii, Wireless ATM for Broadband Multimedia Wireless Access: The ATMmobil Project, IEEE Personal Communications, pp. 6680, October, 1999.

[6] A. Acampora, Wireless ATM: A Perspective on Issues and Prospects, IEEE Personal Communications, pp. 8-17, August, 1996.

[7] D. Raychaudhuri, Wireless ATM Networks: Architecture, System Design and Prototyping, IEEE Personal Communications, pp. 42-49, August, 1996.

[8] J. Mikkonen et alli, Emerging Wireless Broadband Networks, IEEE Communications Magazine, pp. 112-117, February, 1998.

[9] K. Y. Eng et alii, BAHAMA, A Broadband Ad-Hoc Wireless ATM LocalArea Network, Proc. IEEE International Conference on Communications (ICC95), pp. 1216-1223, June, 1995.

[10] F. C. Cheng and J. M. Holtzman, Wireless Intelligent ATM Network and Protocol Design for Future Personal Communication Systems, IEEE Journal on Selected Areas in Communications, Vol. 15, No 7, pp. 12891307, September, 1997.

[11] J. B. Cain and D. N. McGregor, A Recommended Error Control Architecture for ATM Networks with Wireless Links, IEEE Journal on Selected Areas in Communications, Vol. 15, No 1, pp. 16-28, January, 1997.

[12] I. Joe, Error Control for Wireless ATM Networks, PhD Thesis, Georgia Institute of Technology, July, 1998.

[13] C. Schuler, Design and Implementation of an Adaptive Error Control Protocol, GMD Research Series, No 21, 1999.

[14] S. Aikawa, Y. Motoyama and M. Umehira, Error Correction and Error Detection Techniques for Wireless ATM Systems", Wireless Networks, Vol. 3, pp. 285-290, 1997.

[15] J. H. Seo, H. T. Oh and S. W. Seo, A New MAC and DLC Scheme Considering Channel Conditions in Wireless ATM, Proc. Vehicular Technology Conference (VTC 2000), pp. 987-991, Tokyo, Japan, 2000. 
[16] K. Yamada, H. Okada and T. H. Lai, An Integrated Dynamic Error Control Scheme for Wireless ATM Networks, IEICE Transactions on Fundamentals, Vol. E82-A, No 7, pp. 1261-1268, July, 1999.

[17] I. F. Akyildiz, I. Joe, H. Driver and Y. L. Ho, An Adaptive FEC Scheme for Data Traffic in Wireless ATM Networks, IEEE Transactions on Networking, Vol. 9, No 4, pp. 419-426, August, 2001.

[18] P. R. Denz, Performance of Error Control Coding Techniques and the Development of a Dynamic Error Control Coding Mechanism for Wireless ATM, PhD Thesis, Faculty of North Carolina State University, 1999.

[19] M. M. Al-Khatib, New Wireless ATM Automatic Error Control System, $\mathrm{PhD}$ Thesis, University of Southwestern Lousiana, 1999.

[20] I. S. Reed and X. Chen, Error Control Coding for Data Networks, Kluwer Academic Publishers, 1999.

[21] J. G. Proakis, Digital Communications, second edition, McGraw-Hill, 1989.

[22] J. M. C. Brito, Error Control in Wireless ATM Networks, PhD. Thesis, State University of Campinas - Unicamp, Brazil, 2003 (in Portuguese).

[23] J. M. C. Brito and I. S. Bonatti, A Comparison of End-to-End Delay in Wireless ATM Networks using FEC and Selective Repeat ARQ in the Wireless Link, $3^{\text {rd }}$ International Conference on Information, Communications, and Signal Processing (ICICS 2001), 5 pages, Singapore, 15-18 October, 2001.

[24] J. M. C. Brito and I. S. Bonatti, A Comparison of End-to-End Delay in Wireless ATM Networks using FEC and Go-Back-N ARQ in the Wireless Link, $5^{\text {th }}$ IEEE Malaysia International Conference on Communications (MICC 2001), pp 84-89, Kuala Lumpur, Malaysia, 2124 October, 2001.

[25] J. M. C. Brito and I. S. Bonatti, A Simple Adaptive Error Control Scheme for Wireless ATM Networks, International Symposium on Telecommunications (IST 2001), pp. 333-336, Tehran, Iran, 1-3 September, 2001.

[26] J. M. C. Brito and I. S. Bonatti, An Improvement in a Simple Adaptive Error Control Scheme for Wireless ATM Networks, Second IASTED International Conference on Communication Systems and Networks, pp. 205-210, Benalmádena, Spain, 8-10 September, 2003.

[27] M. De Prycker, Asynchronous Transfer Mode (ATM): Solution for Broadband ISBN, 3rd Edition, Ellis Horwood LTD, 1991.

[28] D. Raychaudhuri, L. J. French, R. J. Siracusa, S. K. Biswas, R. Yuan, P. Narasimhan and C. A. Johnston, WATMnet: A Prototype Wireless ATM System for Multimedia Personal Communication, IEEE Journal on Selected Areas in Communications, Vol. 15, No 1, pp. 83-95, January, 1997.

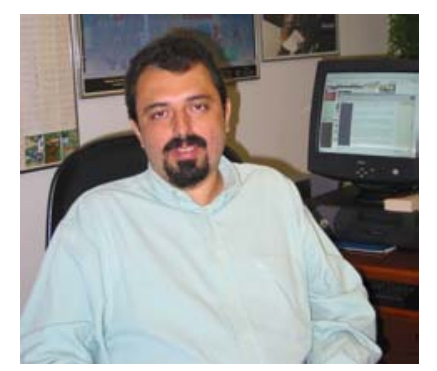

José Marcos C. Brito received his diploma in electrical engineering from the National Institute of Telecommunications (Inatel), Brazil, in 1986, his M. Sc. degree in electrical engineering from State University of Campinas, Brazil, in 1998, and his $\mathrm{Ph} . \mathrm{D}$. degree in electrical engineering from State University of Campinas in 2003. Since 1986 he has been a professor at Inatel, where he has held several management positions, including vice-principal and director for research. Currently he is a Full Professor at Inatel. $\mathrm{He}$ has worked as a consultant for several telecommunications companies in Brazil and has published several papers in international conferences in the telecommunications area. He is the editor-inchief of the Telecommunications Journal edited by Inatel and has served as a program committee member in some international conferences. His current research interests include computer communications networks, multiple access systems, modeling and performance evaluation of communication systems, wireless networks and error control schemes.

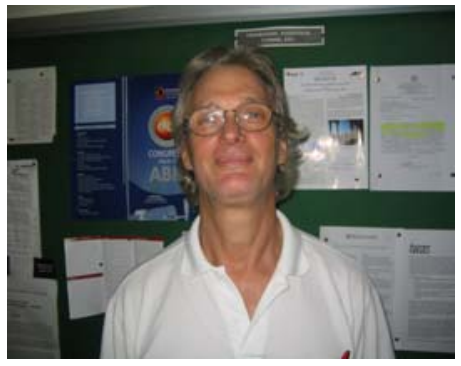

Ivanil S. Bonatti was born in MogiMirim, SP, Brazil, in 1951. He received the B.Sc. (1973) and M.Sc. (1975) degrees in Electrical Engineering from the University of Campinas, UNICAMP, Brazil, and the "Doctorat en Automatique" degree from the University Paul Sabatier, Toulouse, France, in 1981. He joined the School of Electrical and Computer Engineering of the University of Campinas in 1975, where he is currently a Full Professor. His main interests are signal processing, circuit theory, simulation analysis and telecommunication. 